\title{
Thailand-Japan's Volume of Trade Influencing on Socio-economic and Well-Being Development Using Maximum Entropy Bootstrap
}

\author{
Prasert Chaitip, Chukiat Chaiboonsri \\ Chiang Mai University, Chiang Mai, Thailand
}

\begin{abstract}
This paper is preliminary information on some empirical findings based on an analysis of Thailand's macroeconomic data. The purpose of the study is to quantify social-economic well-being development with special emphasis on poverty. The maximum entropy bootstrap was presented that did not require the property of stationarity. Moreover, the methodology was stratified both the ergodic theorem and the central limit theorem. Thailand's GINI index and Thailand-Japan's volume of trade data cover the 14-year period from 1998 to 2011. There is a statistically significant negative non-linear relationship between endogenous Thailand's GINI index and exogenous Thailand-Japan's volume of trade. The results confirmed that every lagged one percent increase in Thailand-Japan's volume of trade influenced by a decrease of Thailand's GINI index coefficient 0.039 percent with the asymmetric around 0 -almost closed to range condition -0.034824348 at $2.5 \%$.
\end{abstract}

Keywords: Thailand-Japan, volume of trade, GINI index, maximum entropy bootstrap, Highest Density Regions (HDR) approach

\section{Introduction}

Poverty reduction has played an important role to enhance Thailand social-economic well-being development in a sufficiency economy. Thailand-Japan's volume of trade amounted influencing on social-economic well-being development. Responsible poverty reduction is fundamental to well-being development. An alternative framework for measuring the relative effects about how Thailand-Japan's volume of trade influences social-economic well-being development is discussed. Thai's history of bilateral relationships between Thailand and Japan was started a long time ago (relations between Ryukyu (Okinawa) and Ayudhaya as far back as the 15th century). The volume of trade between Thailand and Japan for during periods of 2002-2012 also has increased for specified period. Both the Japan and Thailand still had a relationship with each other for a long time ago especially the volume of their trade. The research questions can be defined: Does the Thailand-Japan's volume of trade impact on social-economic well-being development of Thailand? And also does the use of maximum entropy bootstrap as a computer-based econometric procedure help to explain social-economic well-being development? In the study of poverty, the authors investigated the

Prasert Chaitip, Ph.D., Associate Professor, Faculty of Economics, Chiang Mai University.

Chukiat Chaiboonsri, Ph.D., Lecturer, Faculty of Economics, Chiang Mai University.

Correspondence concerning this article should be addressed to Prasert Chaitip Ph.D., Faculty of Economics, Chiang Mai University, Chiang Mai, Thailand. E-mail: pchaiti@hotmail.com. 
influence of the rate of change between total trade volumes between Thailand-Japan on Thailand's GINI index reduction.

Poverty reduction has played an important role to enhance Thailand social-economic well-being development in a sufficiency economy. Thailand-Japan's volume of trade amounted influencing on social-economic well-being development. Responsible poverty reduction is fundamental to the sustainable development. An alternative framework for measuring relative effects about Thailand-Japan's volume of trade influences on social-economic well-being development. Does the use of maximum entropy bootstrap as a computer-based econometric procedure help to explain social-economic well-being development? The authors investigated the influence of Thailand-Japan's volume of trade on Thailand's GINI index reduction during the period of 1998-2011.

\section{Dynamic Econometric Modeling}

An empirical analysis for Thailand based on the maximum entropy bootstrap framework for analyzing a transition to a sustainable economy. Econometric models are often used to forecast economic well-being developments. Since a model was utilized to generate quantitative forecasts of international economic activity, it is of interest to determine as following: Economic indicators of Thailand for sustainable development need to be developed to provide solid phrase. A model can be expressed by $\ln y=\operatorname{lnf}(x 1, x 2)$. The natural $\log$ button on programming software is probably labeled $\ln$. " $\ln$ (gini) denotes the natural logarithm transformation of a number gini as Thailand's GINI index is a function of $\ln (x 1$ or gini, 1 denotes lagged gini by one period and $x 2$ 1t.j 1 denotes lagged aggregate Thailand-Japan's volume of Trade by one period)".

The dynamic form means that GINI as value of Thailand's GINI index depends upon the value of $x 1$ or GINI, 1 (denotes lagged GINI by one period) and $x 2$ (denotes lagged aggregate Thailand-Japan's volume of trade by one period). Value of Thailand's GINI index or GINI can be written in terms of GINI, 1, and 1t.j 1.

Econometric modelling can be adopted to investigate of ambiguity states that the timed behavior of the stochastic process. The maximum entropy experiment has recently introduced a process to fix those problems of ambiguity states especially within the case of econometric model estimation. The maximum entropy bootstrap method can be found in the research work of Vinod (2006) and Vinod and Lopez-de-Lacalle (2009). An overview of the steps in Vinod's ME bootstrap algorithm 6 in seven steps was shown as follows: First step arranges the original data in increasing order to create order statistics $X(t)$ and stores the ordering index vector. The second step computes intermediate points $Z t=(X(t)+X(t-1)) / 2$ for $t=1, t-1$ from the order statistics. The third step computes the trimmed mean mtrm of deviations $X t-X t-1$ among all consecutive observation and also computes the lower limit for left tail as $Z 0=X(1)-$ mtrm and upper limit for right tail as $Z T=X(T)-\mathrm{mtrm}$. These limits become the limiting intermediate point. The fourth step computes the mean of the maximum entropy density within each interval such that the "mean-preserving constraint" (designed to eventually satisfy the ergodic theorem) is satisfied. Interval means are denoted as $m_{t}$. The means for the first and the last interval have simpler formulas. The fifth step generates random numbers from the [0, 1] uniform interval, computing sample quantiles of the ME density at those points and sorting them. The sixth step reorders the sorted sample quantiles by using the ordering index of step. Lastly, the seventh step recovers the time dependence relationships of the original observed data. Repeat steps two to six several times (e.g., 999).

The maximum entropy approach is newly one statistical process to fix those problems especially with the 
estimation of econometric model. And also the maximum entropy bootstrap method can be found in Vinod (2006) and Vinod and Lopez-de-Lacalle's (2009) works. The overview of the steps in Vinod's ME bootstrap algorithm can be found in detail. Moreover, the Highest Density Regions (HDR) approach was conducted to reaffirm an estimation developed by Hyndman (1996).

\section{Results and Discussions}

There comes a time in Thailand as one of Asian developing countries takes a stand to investigate using research that could have a huge influence on stopping this poverty crisis. The relevant data on Thailand's GINI index and Thailand-Japan's volume of trade was collected for the 14-year period of 2006-2010. For Thailand's poverty reduction the study used Gini coefficient as a measure of income inequality. It is an export-oriented economy that Thailand is a sufficiency economy with exports accounting for more than two-thirds of her aggregate demand in terms of GDP. The results of the relationship between Thailand's GINI index and Thailand-Japan's volume of trade were proved. The appropriate correction for the first-footstep estimates is that "simple regression method" results in a loss of some statistical properties suffered from the problems of time series analysis such as stationary or non-stationary and long-memory or short-memory. There was a statistically significant negative non-linear relationship between endogenous Thailand's GINI index and exogenous Thailand-Japan's volume of trade. The results confirmed that every lagged one percent increase in Thailand-Japan's volume of trade is influenced by a decrease of Thailand's GINI index coefficient 0.039 percent (see Table 1).

Table 1

Regression Output for Thailand-Japan's Volume of Trade Influenced Value of Thailand's GINI Index During Period of 1998-2011

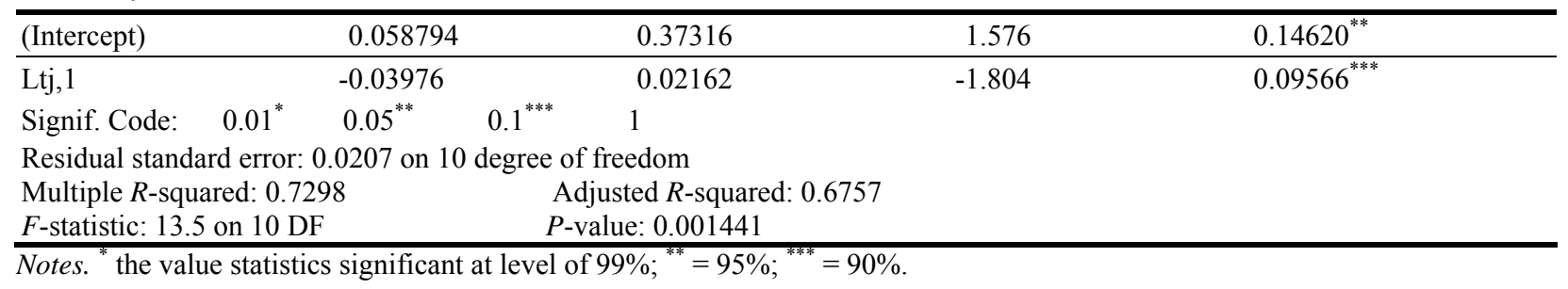

For the second-footstep, a maximum entropy bootstrap technique for time series simply adopted to estimate as testing methods for evaluating estimates. Thailand is the second largest economy in Southeast Asia, after Indonesia. Based on the maximum entropy bootstrap approach statistical methods confirmed that the lagged volume grows one percent of $x 21$ t. j 1 (denotes Thailand-Japan's volume of trade by lagged one period) is associated with Thailand's GINI index that comparatively decrease over time 0.03976 percent (see Table 1). There is a statistically significant negative non-linear relationship between endogenous Thailand's GINI index and exogenous Thailand-Japan's volume of trade. The results confirmed that every lagged one percent increase in Thailand-Japan's volume of trade influenced by a decrease of Thailand's GINI index coefficient 0.039 percent with the asymmetric around 0 -almost closed to range condition 0.034824348 at $2.5 \%$ (see Table 2).

In sum, footsteps on the statistical mountain models are introduced (see Table 2). Researchers need to be aware of statistical investigations to detect the inappropriate correction for the first-footstep estimates. Traditional simple regression method results demonstrate a loss of some statistical properties suffered from the 
problems of time series analysis such as stationary or non-stationary and long-memory or short-memory. The second-footstep and the third-footstep are statistical corrected conditions.

Table 2

Output Bootstrap for Thailand-Japan's Volume of Trade Influenced Value of Thailand's GINI Index During Period of 1998-2011

\begin{tabular}{lll}
\hline & $2.5 \%$ & $97.5 \%$ \\
\hline Simple percentile & -0.003042002 & 0.08165662 \\
Asymmetric around 0 & -0.034824348 & 0.06883638 \\
Boot percentile & -0.003091874 & 0.08219425 \\
Boot norm & 0.075869096 & 0.15645671 \\
Boot basic & 0.064232374 & 0.14951850 \\
\hline
\end{tabular}

Note. Source: From the authors' own computed.

Highest density region of $\beta_{3}$ estimates : Hall model

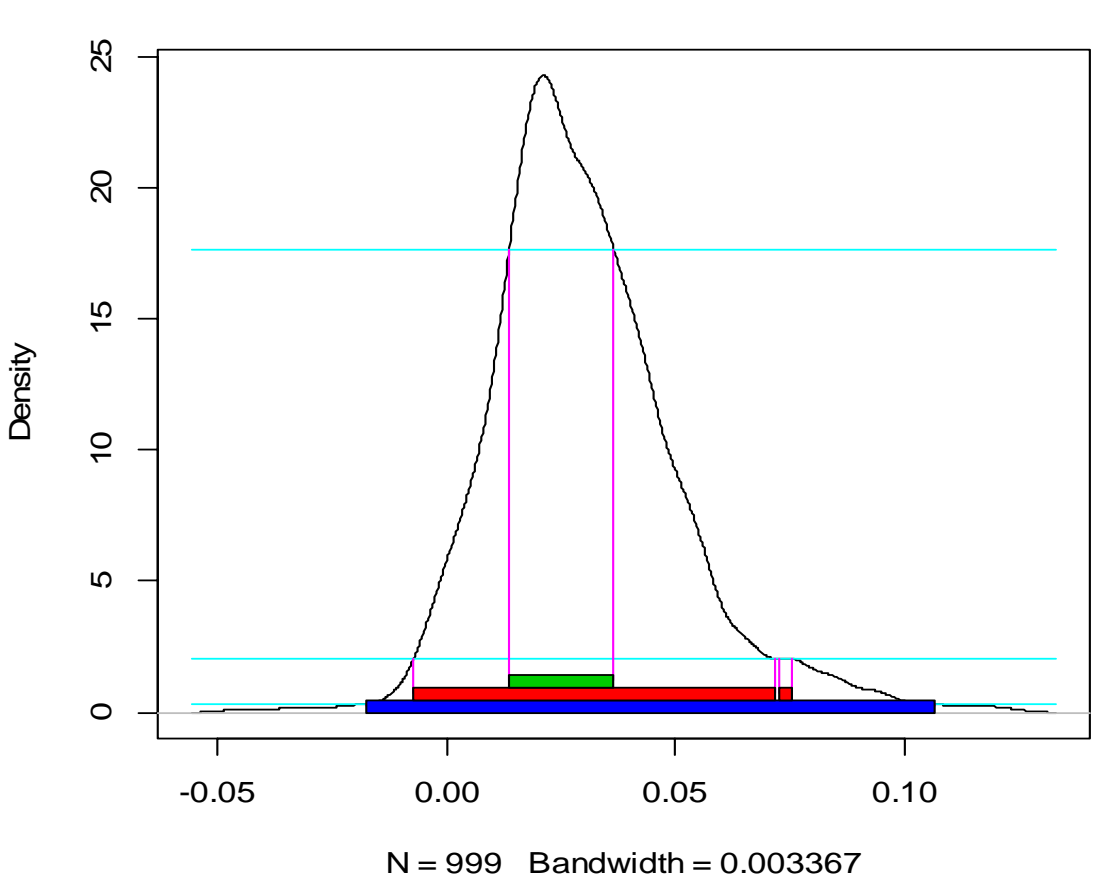

Figure 1. Highest density confidence region for estimates of parameter estimated by function HDR (coefficient of lagged Thailand-Japan's volume of trade). Source: From the authors' own computed.

Moreover, the preliminary testing technique can be used without performing differencing and de-trending methods under all types of non-stationarity including, and fractional integration. This in turn improves reliability in the sense of avoiding specification errors caused by preliminary testing.

Under a framework for causality analysis, the study provided conclusive evidence regarding the causal relationship between Thailand-Japan's volumes of trade influencing value of Thailand's GINI index during the period of 1998-2011. The testing reveals that a statistically significant relationship does exist. In addition, the results once again indicate a causal relationship between the value of Thailand's GINI index and Thailand-Japan's volumes of trade investigated case. Our findings are supported to the time period chosen in 
model specification. The finding indicated the parallel of results that can explain some of the variation in causal conclusions observed in the literature.

The findings supporting the hypothesis provide strong evidence of a huge influence on stopping this poverty crisis in Thailand. Based on the causal relationship of the results, it is possible that some of the previous findings can be observed by rejecting the null of no causality due to the size formations typical for sample statistical inference based on asymptotic theory. Such size formations can be orders of magnitude when bootstrapping is used that is suitable for performing such analysis using time series data. Therefore, to confirmed the maximum entropy bootstrap for time series as testing methods for evaluating estimates the third-footstep proposed HDR-testing method for evaluating statistical output estimates. In Figure 1, the coefficients of Thailand-Japan's volume of trade stay around 90\% of HDR confidence regions depicted on the blue area computed $N=999$. It means that this coefficient is reliably unique and sufficient conditions for the existence non-linear relationship in dynamic form based on the HDR approach (Hyndman, 1996).

Finally, future research should focus on testing the validity of three footsteps diagnosis by extending this analysis to other economic determinants. Potentially fruitful ways for future economic research in addition include using aggregated data to experiment other useful variables.

\section{Conclusions}

The government of Thailand announced the poverty alleviation policy on the national agenda that aims to strengthen Thailand's democratic and always supports Thailand's ongoing poverty reduction programs including health care and education for the needy. Real Thailand-Japan's volume of trade adopted as an economic enhancement of exogenous policy tool to remove crippling poverty by strangling national economic and social well-being development, in this investigation every lagged one percent increase in Thailand-Japan's volume of trade a coefficient 0.039 percent swallowed decrease of Thailand's GINI index or the national poverty. In sum, Thailand social-economic well-being development was influenced by Thailand-Japan's trade volumes especially on issues of poverty reduction. Based on the maximum entropy, bootstrap was proposed in the context of hypothesis testing as a necessary condition in the second-foot step. Also the third-footstep proposed HDR-testing method for evaluating statistical output estimates was confirmed as a sufficient condition. All investigated results are confirmed that there always exists a statistically significant negative non-linear relationship between endogenous Thailand's GINI index and exogenous Thailand-Japan's volumes of trade. The results confirmed that every lagged one percent increase in Thailand-Japan's volume of trade influenced by a decrease of Thailand's GINI index coefficient 0.039 percent with the asymmetric around 0 -almost closed range condition -0.034824348 at $2.5 \%$ introduces a lower bound pronounced non-linearity, closed-form solutions.

\section{References}

Chokethaworn, K., Chaitip, P., \& Chaiboonsri, C. (2012). Frontier of econometrics time series analysis in ICT's stock market of Thailand: Maximum entropy bootstrap approach. Procedia Economics and Finance, 1, 81-87.

Fox, J. (2002). An R and S-PLUS companion to applied regression. Thousand Oaks, C.A.: Sage Publications. Hyndman, R. J. (1996). Computing and graphing highest density regions. The American Statisticians, 50, 120-126.

Vinod, H. D. (2002). Econometric applications of generalized estimating equations for panel data Chaturvedi. Handbook of Applied Econometrics, 26, 553-574. 
Vinod, H. D. (2004). Ranking mutual funds using unconventional utility theory and stochastic dominance. Journal of Empirical Finance, 11(3), 353-377.

Vinod, H. D. (2006). Maximum entropy ensembles for time series inference in economics. Journal of Asian Economics, 17(6), 955-978.

Vinod, H. D. (2008). Hands-on intermediate econometrics using R: Templates for extending dozens of practical examples. New Jersey: World Scientific.

Vinod, H. D., \& Lopez-de-Lacalle, J. (2009). Maximum entropy bootstrap for time series: The meboot R package. Journal of Statistical Software, 29(5), 1-19. 